\title{
Increased Six1 expression is associated with poor prognosis in patients with osteosarcoma
}

\author{
LEMENG CHAO $^{1,2}$, JIANFENG LIU ${ }^{3}$ and DEWEI ZHAO ${ }^{3}$ \\ ${ }^{1}$ Graduate College, Southern Medical University, Guangzhou, Guangdong 510515; ${ }^{2}$ Department of Orthopaedics, Inner \\ Mongolia People's Hospital, Hohhot, Inner Mongolia Autonomous Region 010017; ${ }^{3}$ Department of Orthopaedics, \\ Affiliated Zhongshan Hospital, Dalian University, Dalian, Liaoning 116001, P.R. China
}

Received May 5, 2015; Accepted September 16, 2016

DOI: $10.3892 / \mathrm{ol} .2017 .5803$

\begin{abstract}
Sine oculis homeobox homolog 1 (Six1) is an evolutionarily conserved transcription factor that acts as master regulator of development and is frequently dysregulated in various types of cancer. Six 1 has been demonstrated to be upregulated in human osteosarcoma cell lines compared with osteoblastic cell lines. However, the association of Six 1 expression with the progression and prognosis of osteosarcoma patients remains unclear. The purpose of the present study was to investigate the association between Six 1 expression and the clinicopathological characteristics and prognosis of osteosarcoma. Six1 protein was detected by immunohistochemistry in a series of 100 osteosarcoma patients, and Kaplan-Meier survival analysis was performed to assess prognosis. The results revealed that increased Six1 protein expression was prevalent in osteosarcoma and was significantly associated with Enneking stage $(\mathrm{P}=0.002)$ and tumor size $(\mathrm{P}=0.010)$. Additionally, according to the log-rank test and Cox regression model, expression of Six 1 is indicated to be an independent prognostic factor in osteosarcoma patients. In summary, positive expression of Six 1 protein is closely associated with the tumor progression and poor survival of osteosarcoma patients. The results suggest that Six1 is a overexpressed in individuals with poor prognosis, and may thus be used as a prognostic biomarker in patients with osteosarcoma.
\end{abstract}

\section{Introduction}

Osteosarcoma is the most common primary malignant bone tumor, accounting for $\sim 20 \%$ of primary malignancies of the bone among children and adolescents. It frequently leads to

Correspondence to: Professor Dewei Zhao, Department of Orthopaedics, Affiliated Zhongshan Hospital, Dalian University, 6 Jiefang Street, Dalian, Liaoning 116001, P.R. China

E-mail: ZDW20140101@126.com

Key words: Six 1, immunohistochemistry, osteosarcoma, prognosis lung metastasis and often ultimately results in mortality $(1,2)$. Osteosarcoma is highly aggressive and has a wide range of histological appearances. Conventional osteosarcoma may be classified as osteoblastic, chondroblastic or fibroblastic, depending on the predominant type of extracellular matrix present (3). However, no statistically significant differences have been identified between these three histological types with regard to the survival of patients with high-grade tumors. Furthermore, the treatment for all types is similar (4). The prognosis of this disease is poor despite the use of multimodal therapy, including surgery, radiotherapy and chemotherapy $(5,6)$. Progression to metastatic disease is the predominant cause of treatment failure, while the cellular and molecular mechanisms defining the biological behavior of osteosarcoma cells are poorly understood (6). Therefore, numerous studies have been conducted to broaden the understanding of factors governing the behavior of osteosarcoma cells in order to improve treatment results by identifying potential therapeutic targets (6-8).

Sine oculis homeobox homolog 1 (Six1) is a mammalian homolog of the Drosophila sine oculis gene; it is highly conserved between Drosophila and humans $(9,10)$. The appropriate expression of this gene is crucial for the development of multiple organs, including the brain, eyes, ears, craniofacial structures and kidney sensory structures (11-13). Aside from its involvement in early organ development, Six 1 is often aberrantly expressed in a variety of tumor types, including breast cancer (14), ovarian cancer $(15,16)$, cervical cancer (17,18), Wilms' tumors (19), rhabdomyosarcomas (20) and hepatocellular carcinoma (21). Notably, the aberrant expression of Six1 in cancer can induce developmental programs out of context, which contributes to tumor onset and progression $(22,23)$.

The association between Six1 and the prognosis of osteosarcoma remains unknown. The present study was conducted to investigate the correlation of Six 1 with clinicopathological features in osteosarcoma and its effect on the prognosis of osteosarcoma patients.

\section{Materials and methods}

Patients and tissues. In the present study, resected specimens from 100 osteosarcoma patients who had been 
clinically and pathologically diagnosed between January 2006 and September 2013 were retrieved from the Department of Orthopaedics of Inner Mongolia People's Hospital (Hohhot, Inner Mongolia Autonomous Region, China) for tissue microarray (TMA) construction and immunohistochemical (IHC) analysis. The included patients had undergone preoperative treatment according to a standard protocol, consisting of neoadjuvant chemotherapy [six cycles of cisplatin (300-400 mg/m $\mathrm{m}^{2}$ on days $1-5$ per month) and methotrexate ( $1 \mathrm{~g} / 6 \mathrm{~h}$ on days $1-3$ per month) were administered with a minimum of a 21-day interval], followed by appropriate surgical management and postoperative adjuvant chemotherapy. Detailed pathological and clinical data were collected by reviewing medical records; collected data included age, gender, tumor site, histological type, Enneking stage (24) and tumor size. Two investigators (L.C. and D.Z.) who were blinded to the clinical information confirmed all histological diagnoses and judged staining scores independently. The study protocol was performed under the approval of the Ethics Committee of the Inner Mongolia People's Hospital and informed consent was obtained from all patients. Follow-up was terminated on 31 December 2013. Overall survival (OS) was defined as the interval between surgery and mortality or the last observation taken. The data were censored at the last follow-up period for living patients. Disease-free survival (DFS) was defined as the interval between the date of surgery and the date of diagnosis of any type of relapse or the last follow-up assessment.

TMA construction and IHC analysis. Representative sections of osteosarcoma tissues in the pre-existing paraffin-embedded tissue blocks were determined according to the overlaid hematoxylin and eosin-stained slides. The TMA was constructed using a needle to punch cylinders of $1.5 \mathrm{~mm}$ diameter from the representative section of each block, and placing the cylinders into an array on a recipient paraffin block. Multiple sections (5.0- $\mu$ m-thick) were cut from the TMA block and mounted on microscope slides for IHC analysis. The TMA consisted of a total of 100 cases of paraffin-embedded osteosarcoma tissue. The clinical characteristics of the patients are summarized in Table I. The TMA slide was dried overnight at $37^{\circ} \mathrm{C}$, deparaffinized in xylene, rehydrated through a graded alcohol series, and then immersed in $3 \%$ hydrogen peroxide for $10 \mathrm{~min}$ to inactivate peroxidase activity. Antigen retrieval was performed by microwave heating in citrate buffer $\left(\mathrm{pH} \mathrm{6.0)}\right.$ at $100^{\circ} \mathrm{C}$ for $20 \mathrm{~min}$, then cooling for $20 \mathrm{~min}$ at room temperature to expose antigenic epitopes. The slides were pre-incubated with 5\% normal goat serum (Guangzhou Jetway Biotech Co., Ltd., Guangzhou, China) at room temperature for $30 \mathrm{~min}$ to reduce non-specific reactions. A primary rabbit polyclonal antibody against Six1 (HPA001893; Atlas Antibodies AB, Stockholm, Sweden) was diluted $(1: 1,000)$ with 1 X PBS and applied overnight in a humidified chamber at $4^{\circ} \mathrm{C}$. The slides was sequentially incubated with a goat anti-rabbit polymer peroxidase-labeled secondary antibody (1:500; ZDR-5306; ZSGB-Bio, Beijing, China) for $20 \mathrm{~min}$ at room temperature, and then visualized using 3,3'-diaminobenzidine (Beyotime Institute of Biotechnology, Haimen, China) as a chromogen. Finally, the sections were counterstained with hematoxylin.
A known Six1-positive slide was used as a positive control, and PBS replaced anti-Six 1 primary antibody as the negative control.

Evaluation of IHC staining. Immunoreactivity for the Six1 protein was scored according to the staining intensity and positive percentage. Sections were considered positive if they exhibited immunoreactivity in the nucleus and/or cytoplasm when observed by an evaluator who was blinded to the clinical history and outcome of the case. Positive percentage scores were assigned according to the following scale: $0,0 \%$ positively stained cells; $1,1-25 \%$ positively stained cells; $2,26-50 \%$ positively stained cells; and $3,>50 \%$ positively stained cells. Staining intensity was also scored semi-quantitatively as follows: 0 , none; 1 , mild; 2 , moderate; and 3, intense. A total score was obtained (ranging from 0 to 9 ) by multiplying the positive percentage score and the intensity score for each section. From the total scores, 0, 1-3, 4-6, and 7-9 were recorded as,,-+++ , and +++ , respectively. Negative expression was defined as a score of 0 or 1 ; positive expression was defined as a score of $>1$. The scores were accepted if the two investigators (L.C. and D.Z) agreed with the values. Otherwise, the values were re-estimated until a consensus was reached. The conclusions were in complete agreement in $80 \%$ of the cases, which indicated that the scoring method was highly reproducible.

Statistical analysis. Statistical analysis was performed using the SPSS statistical software program (version 18.0; SPSS, Inc., Chicago, IL, USA). The associations between Six1 protein expression and the clinicopathological data of osteosarcoma patients were analyzed using the $\chi^{2}$ test. The association between survival and each variable was determined with the Kaplan-Meier method. Differences between survival rates were analyzed with the log-rank test and Cox regression analysis. $\mathrm{P}<0.05$ (two-tailed) denotes the presence of a statistically significant difference.

\section{Results}

Clinicopathological data of the patients. As shown in Table I, among the 100 studied cases, 35 (35\%) were female and $65(65 \%)$ were male, equating to a female:male ratio of $\sim 1: 2$. The age ranged from 7 to 69 years, with a mean \pm standard deviation of $24.70 \pm 13.66$ years; 62 patients $(62 \%)$ were aged $\leq 25$ years and 38 patients $(38 \%)$ were aged $>25$ years. Regarding the anatomical site of the tumor, 39 tumors $(39 \%)$ were in the femur, $15(15 \%)$ were located in the tibia, and 5 tumors $(5 \%)$ were located in the humerus; the remaining 41 tumors $(41 \%)$ were located in other sites. The size of the tumors ranged from 2 to $17 \mathrm{~cm}$, with a mean of $8.49 \pm 3.54 \mathrm{~cm}$.

Sixl expression in the osteosarcoma tissues. IHC staining was performed to investigate Six1 protein expression levels. The analysis revealed that the expression of Six 1 was predominantly localized in the cytoplasm and the distribution of positive cells was primarily located in peripheral portion of the osteosarcoma; a number of osteosarcoma tissues exhibited strong staining, while others exhibited weak or absent 
Table I. Six 1 expression in relation to clinical and pathological factors in a series of 100 patients with osteosarcoma.

\begin{tabular}{|c|c|c|c|c|c|}
\hline \multirow[b]{2}{*}{ Variable } & \multirow[b]{2}{*}{ Number } & \multicolumn{2}{|c|}{ Six 1 expression } & \multirow[b]{2}{*}{$\chi^{2}$} & \multirow[b]{2}{*}{ P-value } \\
\hline & & Negative & Positive & & \\
\hline Age (years) & & & & & 0.798 \\
\hline$\leq 25$ & 62 & 31 & 31 & 0.065 & \\
\hline$>25$ & 38 & 20 & 18 & & \\
\hline Gender & & & & & 0.367 \\
\hline Female & 35 & 20 & 15 & 0.813 & \\
\hline Male & 65 & 31 & 34 & & \\
\hline Tumor site & & & & & 0.085 \\
\hline Femur & 60 & 32 & 28 & 4.924 & \\
\hline Tibia & 27 & 16 & 11 & & \\
\hline Humerus & 13 & 3 & 10 & & \\
\hline Histological type & & & & & 0.181 \\
\hline Osteoblastic & 49 & 20 & 29 & 4.875 & \\
\hline Chondroblastic & 28 & 16 & 12 & & \\
\hline Fibroblastic & 18 & 11 & 7 & & \\
\hline Telangiectatic & 5 & 4 & 1 & & \\
\hline Enneking stage & & & & & 0.002 \\
\hline I & 18 & 15 & 3 & 9.183 & \\
\hline II & 82 & 36 & 46 & & \\
\hline Tumor size (cm) & & & & & 0.002 \\
\hline$\leq 5$ & 21 & 17 & 4 & 9.543 & \\
\hline$>5$ & 79 & 34 & 45 & & \\
\hline
\end{tabular}

Six 1, sine oculis homeobox homolog 1 .


Figure 1. Immunohistochemical staining of Six 1 protein in osteosarcoma tissues. Six 1 was predominantly expressed in the cytoplasm of osteosarcoma cells. (A and B) Negative Six1 expression: (A) magnification, x100; (B) magnification, x400. (C and D) Positive expression of Six1: (C) magnification, x100; (D) magnification, $\mathrm{x} 400$. Six 1 , sine oculis homeobox homolog 1. 
Table II. Univariate survival analysis of osteosarcoma according to immunohistochemistry scores for Six1 and other clinicopathological factors.

\begin{tabular}{|c|c|c|c|c|c|c|}
\hline \multirow[b]{2}{*}{ Variable } & \multicolumn{3}{|c|}{ Overall survival time (months) } & \multicolumn{3}{|c|}{ Disease-free survival time (months) } \\
\hline & Mean & $95 \% \mathrm{CI}$ & P-value & Mean & $95 \% \mathrm{CI}$ & P-value \\
\hline Six 1 & & & $<0.001$ & & & $<0.001$ \\
\hline Negative & 50.395 & $43.425-57.336$ & & 46.644 & $40.289-52.999$ & \\
\hline Positive & 29.668 & $25.286-34.050$ & & 28.006 & 24.272-31.739 & \\
\hline Age (years) & & & 0.639 & & & 0.938 \\
\hline$\leq 25$ & 42.371 & $35.656-49.085$ & & 38.016 & $32.222-43.809$ & \\
\hline$>25$ & 38.910 & $32.278-45.542$ & & 37.312 & $31.406-43.218$ & \\
\hline Gender & & & 0.273 & & & 0.540 \\
\hline Male & 39.297 & $33.346-45.248$ & & 36.902 & $31.554-42.249$ & \\
\hline Female & 43.978 & $35.821-52.135$ & & 39.065 & $32.221-45.909$ & \\
\hline Tumor site & & & 0.681 & & & 0.223 \\
\hline Femur & 39.627 & $34.000-45.254$ & & 36.434 & $31.723-41.145$ & \\
\hline Tibia & 46.253 & $36.284-56.222$ & & 43.268 & $34.076-52.460$ & \\
\hline Humerus & 31.856 & $22.043-41.133$ & & 29.558 & $20.185-38.931$ & \\
\hline Histologic type & & & 0.790 & & & 0.505 \\
\hline Osteoblastic & 40.786 & $33.788-47.784$ & & 37.098 & $31.145-43.051$ & \\
\hline Chondroblastic & 43.759 & $35.156-52.362$ & & 41.415 & $33.524-49.307$ & \\
\hline Fibroblastic & 37.944 & $26.547-49.342$ & & 36.132 & $25.878-46.386$ & \\
\hline Telangiectatic & 31.800 & $16.850-46.750$ & & 28.000 & $15.298-40.702$ & \\
\hline Enneking stage & & & $<0.001$ & & & $<0.001$ \\
\hline I & 66.750 & $56.684-76.816$ & & 61.185 & $51.637-70.733$ & \\
\hline II & 34.340 & $30.053-38.627$ & & 32.157 & $28.454-35.859$ & \\
\hline Tumor size (cm) & & & $<0.001$ & & & 0.001 \\
\hline$\leq 5$ & 65.059 & $53.404-76.714$ & & 56.588 & $45.940-67.237$ & \\
\hline$>5$ & 35.314 & $30.938-39.690$ & & 33.529 & $29.524-37.535$ & \\
\hline
\end{tabular}

$\mathrm{P}<0.05$ was considered to indicate a statistically significant difference. Six 1, sine oculis homeobox homolog 1; CI, confidence interval.

Table III. Multivariate analysis of factors associated with OS and DFS.

\begin{tabular}{|c|c|c|c|c|c|c|}
\hline \multirow[b]{2}{*}{ Variable } & \multicolumn{3}{|c|}{ OS } & \multicolumn{3}{|c|}{ DFS } \\
\hline & HR & $95 \% \mathrm{CI}$ & P-value & HR & $95 \% \mathrm{CI}$ & P-value \\
\hline Six1 (negative vs. positive) & 1.757 & $1.089-2.835$ & 0.021 & 1.848 & $1.165-2.931$ & 0.009 \\
\hline Gender (female vs. male) & 0.953 & $0.579-1.568$ & 0.849 & 0.885 & $0.554-1.411$ & 0.607 \\
\hline Enneking stage (I vs. II) & 3.126 & $1.432-6.826$ & 0.004 & 2.783 & $1.399-5.537$ & 0.004 \\
\hline Tumor size ( $\leq 5$ vs. $>5 \mathrm{~cm})$ & 2.744 & $1.190-6.328$ & 0.018 & 1.854 & $0.932-3.686$ & 0.078 \\
\hline
\end{tabular}

$\mathrm{P}<0.05$ was considered to indicate a statistically significant difference. OS, overall survival; DFS, disease free survival; HR, hazard ratio; CI, confidence interval; Six 1, sine oculis homeobox homolog 1.

staining (Fig. 1). Positive Six1 expression was observed in $49.0 \%$ of the 100 osteosarcoma tissues. A significant differences in the distribution of IHC scores between the different Enneking stages $(\mathrm{P}=0.002)$ was noted: Positive Six 1 expression was observed significantly more often in cases with high-stage disease compared with cases with a low-stage. A highly significant association was also identified between Six1 expression and tumor size $(\mathrm{P}=0.010)$. However, the other clinicopathological parameters, including age, gender, tumor site and histological type were not significantly associated with Six1 expression (Table I).

Sixl expression is associated with patient prognosis. To evaluate the correlation of Six1 protein expression with 

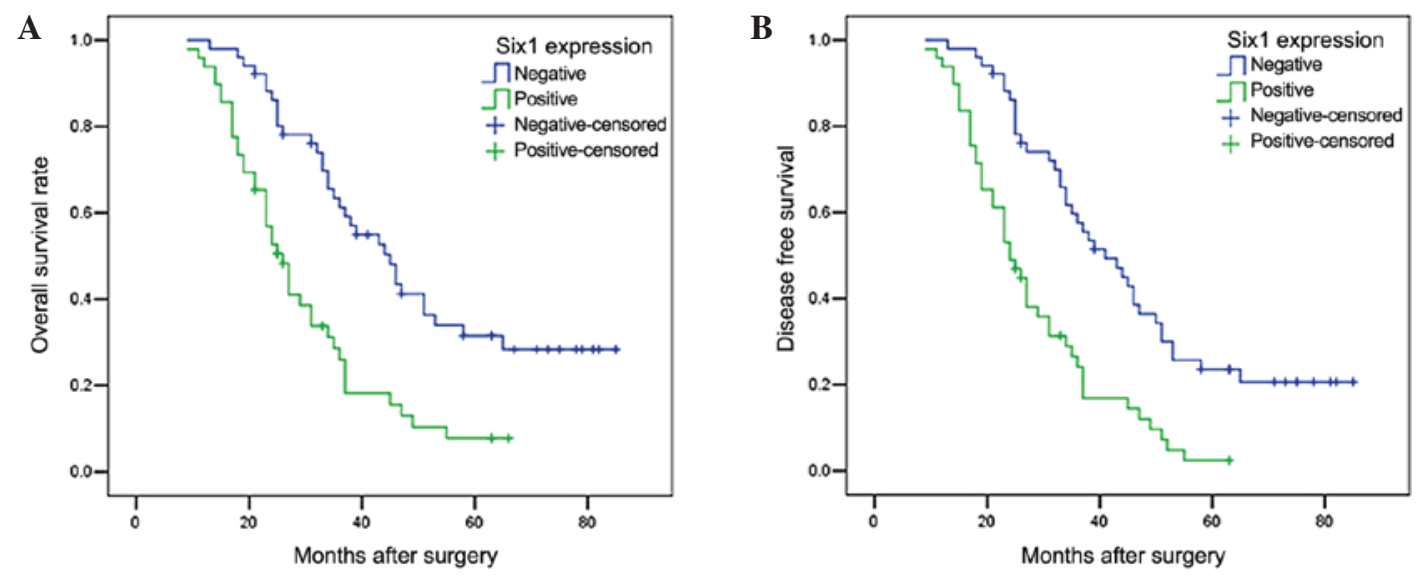

Figure 2. Kaplan-Meier analysis of (A) OS and (B) DFS of patients with osteosarcoma based on positive or negative Six1 expression. Osteosarcoma patients with Six1-positive expression exhibited significantly poorer OS and DFS rates compared with those with Six1-negative expression. OS, overall survival; DFS, disease-free survival; Six1, sine oculis homeobox homolog 1 .

patient prognosis, the osteosarcoma patients were divided into two groups: Negative Six 1 expression $(\mathrm{n}=51)$ and positive Six 1 expression $(n=49)$. A log-rank test and Kaplan-Meier analysis were performed to assess the effect of Six 1 expression on patient survival. The log-rank test revealed that the OS time of osteosarcoma patients with Six1-positive expression was markedly shorter than those with Six1-negative expression $(\mathrm{P}<0.001$; Fig. 2A; Table II). Similar results were also observed in the DFS analysis $(\mathrm{P}<0.001$; Fig. $2 \mathrm{~B}$; Table II). At time of last follow-up, 41 patients in the Six1-positive group had succumbed to disease, with a mean OS time of 29.67 months. Of these, 3 patients developed local recurrence and 6 had lung metastasis. Comparatively, 33 patients succumbed to disease in the Six1-negative group and the mean OS time was 50.40 months. Furthermore, as shown in Table III, multivariate Cox analysis indicated that Six1 expression was one of the independent prognostic factors, along with Enneking stage and tumor size.

\section{Discussion}

Homeobox genes encode transcription factors that are essential for the development of numerous organs and the control of various processes, including cell proliferation, apoptosis, migration and invasion $(11,23,25,26)$. The Six 1 homeoprotein, a member of the Six family of homeodomain transcription factors, has been found to be aberrantly expressed in multiple types of cancer, including breast cancer $(14,22,27)$, hepatocellular carcinomas (21), ovarian cancer (15), Wilms' tumors (19) and rhabdomyosarcomas $(20,28,29)$. Six 1 is also involved in cellular migration and invasion during embryogenesis $(25,30-33)$ and in breast cancer $(34,35)$. A previous study demonstrated that Six 1 was able to promote metastasis by coordinating with transforming growth factor $\beta$ (TGF- $\beta$ ) to increase the expression of vascular endothelial growth factor C (VEGF-C) through two pathways. Firstly, Six 1 is able to enhance TGF- $\beta$ signaling by upregulating TGF- $\beta$ receptor 1 expression, which promotes the activation of SMAD2/3 and its binding to the VEGF-C promoter, thus increasing the expression of VEGF-C. Secondly, Six 1 can cooperate with SMAD2/3 to bind to the VEGF-C promoter and modulate VEGF-C expression. In tumor cells lacking Six 1 expression, the expression of VEGF-C was slightly influenced by TGF- $\beta$ stimulation; however, SMAD2/3 could be phosphorylated and bind to the VEGF-C promoter. Therefore, Six 1 is necessary for TGF- $\beta$ to induce the increased expression of the VEGF-C gene (36). Overexpression of Six1 also significantly enhances the activation of the cyclin D1 promoter in pancreatic cancer and promotes cell cycle progression and proliferation (37). Six1 overexpression is positively correlated with OS and DFS rates of patients with breast cancer (38).

It has been reported that Six1 expression levels are upregulated in osteosarcoma cell lines compared with noncancerous human osteoblastic cell lines, and Six1 was able to increase the growth, proliferation and migration of U2OS cells, as well as suppress apoptosis (39). Therefore, we hypothesized that Six 1 may be associated with the progression and prognosis of osteosarcoma.

In the present study, the expression of Six 1 protein was detected in osteosarcoma samples from cases with various clinicopathological features. Six1 expression was identified to be positively associated with Enneking stage and tumor size, but not age, gender, tumor site or histological type. These results indicate that Six 1 expression in osteosarcoma is associated with osteosarcoma progression. In order to investigate the influence of Six 1 on the prognosis of osteosarcoma patients, 100 patients were followed up after surgery. Six1 was identified as an independent factor able to predict the OS time of osteosarcoma patients. Firstly, the log-rank test revealed that patients with Six1-positive expression tended to have a significantly shorter OS time (mean, 29.67 months) compared with the Six1-negative group (mean, 50.40 months). Secondly, Cox regression analysis identified that Six 1 may act as an independent prognostic factor, in addition to Enneking stage and tumor size.

In summary, Six 1 is differently expressed in cases of osteosarcomas with different clinicopathological features, showing positive correlations with Enneking stage and tumor size, which indicates poor prognosis in patients with positive Six1 expression. Six 1 protein may therefore be suggested as a useful prognostic biomarker for osteosarcoma. 


\section{References}

1. Marina N, Gebhardt M, Teot L and Gorlick R: Biology and therapeutic advances for pediatric osteosarcoma. Oncologist 9: 422-441, 2004

2. Fagioli F, Biasin E, Mereuta OM, Muraro M, Luksch R, Ferrari S, Aglietta M and Madon E: Poor prognosis osteosarcoma: New therapeutic approach. Bone Marrow Transplant 41 (Suppl 2): S131-S134, 2008.

3. Fletcher CDM, Unni KK and Mertens F (eds): Conventional osteosarcoma. In: World Health Organization Classification of Tumours - Pathology and Genetics of Tumors of Soft Tissue and Bone. 4th edition. IARC Press, Lyon, p19, 2013.

4. Chou AJ and Gorlick R: Chemotherapy resistance in osteosarcoma: Current challenges and future directions. Expert Rev Anticancer Ther 6: 1075-1085, 2006.

5. Yao C, Wei JJ, Wang ZY, Ding HM, Li D, Yan SC, Yang YJ and Gu ZP: Perifosine induces cell apoptosis in human osteosarcoma cells: New implication for osteosarcoma therapy? Cell Biochem Biophys 65: 217-227, 2013.

6. Poletajew S, Fus L and Wasiutyński A: Current concepts on pathogenesis and biology of metastatic osteosarcoma tumors. Ortop Traumatol Rehabil 13: 537-545, 2011 (In English, Polish).

7. Hu F, Wang W, Zhou HC and Shang XF: High expression of periostin is dramatically associated with metastatic potential and poor prognosis of patients with osteosarcoma. World J Surg Oncol 12: 287, 2014

8. Nada OH, Ahmed NS and Abou Gabal HH: Prognostic significance of HLA EMR8-5 immunohistochemically analyzed expression in osteosarcoma. Diagn Pathol 9: 72, 2014.

9. Kumar JP: The sine oculis homeobox (SIX) family of transcription factors as regulators of development and disease. Cell Mol Life Sci 66: 565-583, 2009.

10. Anderson AM, Weasner BM, Weasner BP and Kumar JP: Dual transcriptional activities of SIX proteins define their roles in normal and ectopic eye development. Development 139: 991-1000, 2012.

11. Xu PX, Zheng W, Huang L, Maire P, Laclef C and Silvius D: Six1 is required for the early organogenesis of mammalian kidney. Development 130: 3085-3094, 2003.

12. Laclef C, Souil E, Demignon J and Maire P: Thymus, kidney and craniofacial abnormalities in Six 1 deficient mice. Mech Dev 120: 669-679, 2003.

13. Konishi Y, Ikeda K, Iwakura Y and Kawakami K: Six1 and Six4 promote survival of sensory neurons during early trigeminal gangliogenesis. Brain Res 1116: 93-102, 2006.

14. Reichenberger KJ, Coletta RD, Schulte AP, Varella-Garcia M and Ford HL: Gene amplification is a mechanism of Six1 overexpression in breast cancer. Cancer Res 65: 2668-2675, 2005.

15. Behbakht K, Qamar L, Aldridge CS, Coletta RD, Davidson SA, Thorburn A and Ford HL: Six 1 overexpression in ovarian carcinoma causes resistance to TRAIL-mediated apoptosis and is associated with poor survival. Cancer Res 67: 3036-3042, 2007.

16. Imam JS, Buddavarapu K, Lee-Chang JS, Ganapathy S, Camosy C, Chen Y and Rao MK: MicroRNA-185 suppresses tumor growth and progression by targeting the Six1 oncogene in human cancers. Oncogene 29: 4971-4979, 2010.

17. Tan J, Zhang C and Qian J: Expression and significance of Six1 and Ezrin in cervical cancer tissue. Tumour Biol 32: 1241-1247, 2011.

18. Zheng XH, Liang PH, Guo JX, Zheng YR, Han J, Yu LL, Zhou YG and Li L: Expression and clinical implications of homeobox gene Six1 in cervical cancer cell lines and cervical epithelial tissues. Int J Gynecol Cancer 20: 1587-1592, 2010.

19. Li CM, Guo M, Borczuk A, Powell CA, Wei M, Thaker HM, Friedman R, Klein U and Tycko B: Gene expression in Wilms tumor mimics the earliest committed stage in the metanephric mesenchymal-epithelial transition. Am J Pathol 160: 2181-2190,2002

20. Yu Y, Khan J, Khanna C, Helman L, Meltzer PS and Merlino G: Expression profiling identifies the cytoskeletal organizer ezrin and the developmental homeoprotein Six-1 as key metastatic regulators. Nat Med 10: 175-181, 2004.

21. Ng KT, Man K, Sun CK, Lee TK, Poon RT, Lo CM and Fan ST: Clinicopathological significance of homeoprotein Six1 in hepatocellular carcinoma. Br J Cancer 95: 1050-1055, 2006
22. Coletta RD, Christensen K, Reichenberger KJ, Lamb J, Micomonaco D, Huang L, Wolf DM, Müller-Tidow C, Golub TR, Kawakami K and Ford HL: The Six1 homeoprotein stimulates tumorigenesis by reactivation of cyclin A1. Proc Natl Acad Sci USA 101: 6478-6483, 2004.

23. Coletta RD, Christensen KL, Micalizzi DS, Jedlicka P, Varella-Garcia M and Ford HL: Six1 overexpression in mammary cells induces genomic instability and is sufficient for malignant transformation. Cancer Res 68: 2204-2213, 2008.

24. Kundu ZS: Classification, imaging, biopsy and staging of osteosarcoma. Indian J Orthop 48: 328-346, 2014.

25. Zheng W, Huang L, Wei ZB, Silvius D, Tang B and Xu PX: The role of Six 1 in mammalian auditory system development. Development 130: 3989-4000, 2003

26. Ikeda K, Kageyama R, Suzuki Y and Kawakami K: Six1 is indispensable for production of functional progenitor cells during olfactory epithelial development. Int J Dev Biol 54: 1453-1464, 2010

27. Ford HL, Kabingu EN, Bump EA, Mutter GL and Pardee AB: Abrogation of the G2 cell cycle checkpoint associated with overexpression of HSIX1: A possible mechanism of breast carcinogenesis. Proc Natl Acad Sci USA 95: 12608-12613, 1998.

28. Khan J, Bittner ML, Saal LH, Teichmann U, Azorsa DO, Gooden GC, Pavan WJ, Trent JM and Meltzer PS: cDNA microarrays detect activation of a myogenic transcription program by the PAX3-FKHR fusion oncogene. Proc Natl Acad Sci USA 96: 13264-13269, 1999.

29. Yu Y, Davicioni E, Triche TJ and Merlino G: The homeoprotein six1 transcriptionally activates multiple protumorigenic genes but requires ezrin to promote metastasis. Cancer Res 66: 1982-1989, 2006.

30. Ozaki H, Nakamura K, Funahashi J, Ikeda K, Yamada G, Tokano H, Okamura HO, Kitamura K, Muto S, Kotaki H, et al: Six 1 controls patterning of the mouse otic vesicle. Development 131: 551-562, 2004

31. Li X, Oghi KA, Zhang J, Krones A, Bush KT, Glass CK, Nigam SK, Aggarwal AK, Maas R, Rose DW and Rosenfeld MG: Eya protein phosphatase activity regulates Six1-Dach-Eya transcriptional effects in mammalian organogenesis. Nature 426 : 247-254, 2003

32. Grifone R, Demignon J, Houbron C, Souil E, Niro C, Seller MJ, Hamard G and Maire P: Six 1 and Six 4 homeoproteins are required for Pax3 and Mrf expression during myogenesis in the mouse embryo. Development 132: 2235-2249, 2005.

33. Ikeda K, Ookawara S, Sato S, Ando Z, Kageyama R and Kawakami K: Six1 is essential for early neurogenesis in the development of olfactory epithelium. Dev Biol 311: 53-68, 2007.

34. Micalizzi DS, Christensen KL, Jedlicka P, Coletta RD, Barón AE, Harrell JC, Horwitz KB, Billheimer D, Heichman KA, Welm AL, et al: The Six1 homeoprotein induces human mammary carcinoma cells to undergo epithelial-mesenchymal transition and metastasis in mice through increasing TGF-beta signaling. J Clin Invest 119: 2678-2690, 2009.

35. Micalizzi DS, Wang CA, Farabaugh SM, Schiemann WP and Ford HL: Homeoprotein Six 1 increases TGF-beta type I receptor and converts TGF-beta signaling from suppressive to supportive for tumor growth. Cancer Res 70: 10371-10380, 2010.

36. Liu D, Li L, Zhang XX, Wan DY, Xi BX, Hu Z, Ding WC, Zhu D, Wang XL, Wang W, et al: SIX1 promotes tumor lymphangiogenesis by coordinating TGF $\beta$ signals that increase expression of VEGF-C. Cancer Res 74: 5597-5607, 2014.

37. Li Z, Tian T, Lv F, Chang Y, Wang X, Zhang L, Li X, Li L, Ma W, Wu J and Zhang M: Six 1 promotes proliferation of pancreatic cancer cells via upregulation of cyclin D1 expression. PLoS One 8: e59203, 2013

38. Jin H, Cui M, Kong J, Cui X, Lin Z, Wu Q and Liu S: Sineoculis homeobox homolog 1 protein is associated with breast cancer progression and survival outcome. Exp Mol Pathol 97: 247-252, 2014

39. Hua L, Fan L, Aichun W, Yongjin Z, Qingqing C and Xiaojian W: Inhibition of Six1 promotes apoptosis, suppresses proliferation, and migration of osteosarcoma cells. Tumour Biol 35: $1925-1931,2014$ 